\title{
Distribution of Geometrically Weighted Sum of Bernoulli Random Variables
}

\author{
Deepesh Bhati ${ }^{1}$, Phazamile Kgosi ${ }^{2}$, Ranganath Narayanacharya Rattihalli ${ }^{1}$ \\ ${ }^{1}$ Department of Statistics, Central University of Rajasthan, Kishangarh, India \\ ${ }^{2}$ Department of Statistics, University of Botswana, Gaborone, Botswana \\ E-mail: dipesh089@gmail.com, KGOSIPM@mopipi.ub.bw,rnr5@rediffmail.com \\ Received July 6, 2011; revised October 5, 2011; accepted October 13, 2011
}

\begin{abstract}
A new class of distributions over $(0,1)$ is obtained by considering geometrically weighted sum of independent identically distributed (i.i.d.) Bernoulli random variables. An expression for the distribution function (d.f.) is derived and some properties are established. This class of distributions includes $U(0,1)$ distribution.
\end{abstract}

Keywords: Binary Representation, Probability Mass Function, Distribution Function, Characteristic Function

\section{Introduction}

Uniform distribution plays an important role in Statistics. The existence of uniform random variable (r.v.) over the interval $(0,1)$, using $B(1,1 / 2)$ r.v.s is indicated in [1]. As a generalization, in this paper we consider the following geometrically weighted sum of i.i.d. Bernoulli r.v.s

$$
X=\sum_{j=1}^{\infty}\left(\frac{1}{2}\right)^{j} Z_{j},
$$

where $Z_{j}^{\prime} s$ are i.i.d. $B(1, p)$ r.v's. The remainder of the paper is organized as follows. In Section 2 we obtain the characteristic function of $X$ and give an interpretation for the variable $X$. In Section 3 we derive the distribution function of $X$ and prove some of its properties. In Section 4 we discuss the existence of the density function. In Section 5 distribution of sum of a finite number of variables is considered and the graphs of its probability mass function (p.m.f.) and distribution function (d.f.) are given in the Appendix.

\section{The Characteristic Function and an Application of the Model}

\subsection{The Characteristic Function}

Let $F(t)=P\{X \leq t\}$ be the d.f. of $X$. Then by the definition of $X$ we have

$$
\begin{aligned}
F(t) & =P\left(X \leq t \mid Z_{1}=0\right) P\left(Z_{1}=0\right)+P\left(X \leq t \mid Z_{1}=1\right) P\left(Z_{1}=1\right) \\
& =P\left(\frac{X}{2} \leq t\right)(1-p)+P\left(\frac{1}{2}+\frac{X}{2} \leq t\right) p .
\end{aligned}
$$

Hence the characteristic function (c.f.) $\Psi(t)$ of $X$ satisfies the equation

$$
\Psi(t)=(1-p) \Psi(t / 2)+p \mathrm{e}^{i t / 2} \Psi(t / 2) .
$$

That is,

$$
\Psi(t)=\Psi(t / 2)\left[1-p+p \mathrm{e}^{i t / 2}\right] .
$$

Repeating this and replacing $t$ by $t / 2$ each time, we get, for $n=1,2, \cdots$.

$$
\Psi(t)=\Psi\left(t / 2^{n}\right) \prod_{k=1}^{n}\left[1-p+p \mathrm{e}^{i t / 2^{k}}\right] .
$$

The reproductive property of the characteristic function exhibited by (3) is comparable to the characteristic function of an infinitely divisible distribution. For details one may refer to Section 7 of [2].

Since $\Psi\left(t / 2^{n}\right) \rightarrow 1$ as $n \rightarrow \infty$ we have

$$
\Psi(t)=\prod_{k=1}^{\infty}\left[1-p+p \mathrm{e}^{i t / 2^{k}}\right] .
$$

Note that if $p=0$, this infinite product is 1 , and, if $p=$ 1 , the infinite product is $\mathrm{e}^{i t}$. Thus $p=0$ results in $X$ being degenerate at 0 while $p=1$ implies that $X$ is degenerate at 1 . If $p=1 / 2$, then the product term in (4) is

$$
\frac{1}{2^{n}} \frac{\mathrm{e}^{i t}-1}{\mathrm{e}^{i t / 2^{n}}-1} \rightarrow \frac{\mathrm{e}^{i t}-1}{i t} \text { as } n \rightarrow \infty
$$

Thus if $p=1 / 2$ then $X$ has $U(0,1)$ distribution.

\subsection{An Application}

This resulting distribution can be used as a model in a 
situation similar to the following. Suppose a particle has linear movement on the interval $[0,1]$. To capture the particle suppose the following binary capturing technique of dividing the existing interval into two equal halves is used. Suppose initially there are two barriers put at 0 and 1. After one unit of time a barrier is put at the midpoint of 0 and 1. Further the interval in which the particle is found is divided into two equal halves by placing a barrier at their midpoint and the process is continued. The intervals containing the particle keep on shrinking and finally shrink to $X$, the point at which the particle is captured in the long run. The behavior of the particle is known only to the extent that at the moment of placing a barrier after exactly one unit of time the particle is on the right side of the inserted barrier with probability $p$.

\section{Main Results}

\subsection{Notation}

It is known that every number $t, 0 \leq t \leq 1$ has a binary representation through $\left\{a_{n}\right\}_{1}^{\infty}, a_{n}=0$ or 1 , as

$t=\sum_{i=1}^{\infty} a_{i}\left(\frac{1}{2}\right)^{i}$. If a number $t$ has the representation $t=\sum_{i=1}^{k} a_{i}\left(\frac{1}{2}\right)^{i}, a_{k}=1$, we refer $t$ as a finite binary terminating number and such a number can be represented by $r 2^{-k}$, for some $r=1,2, \cdots, 2^{k}-1$. However such a number also can be represented as

$$
\begin{aligned}
& \sum_{i=1}^{\infty} b_{i}\left(\frac{1}{2}\right)^{i}, b_{i}=a_{i}, i=1,2, \cdots, k-1, \\
& b_{k}=0, b_{i}=1, i=k+1, k+2, \cdots
\end{aligned}
$$

It is to be noted that the right tail of the sequence $\left\{a_{i}\right\}$ is of the form $(0,0,0 \cdots)$ while that of the sequence $\left\{b_{i}\right\}$ is of the form $(1,1,1, \cdots)$. In the following as a matter of convention we do not consider representation with the right tail of the form $(1,1,1, \cdots)$. Under such a convention, $t \in[0,1]$ corresponds to a unique binary sequence $\left\{a_{i}\right\}_{1}^{\infty}$ and conversely. If $t=\sum_{i=1}^{\infty} a_{i}\left(\frac{1}{2}\right)^{i}$, then we shall denote this relation as $\stackrel{B R}{\Leftrightarrow}\left\{a_{n}\right\}$, (BR to mean the binary representation).

\subsection{Properties}

\section{Theorem 1:}

Let $t \stackrel{B R}{\Leftrightarrow}\left\{a_{n}\right\}$ and $s_{k}=\sum_{j=1}^{k} a_{j}, k=1,2, \cdots, s_{0}=0$. Then the d.f. of $X$ defined in (1) is given by

$$
F(t)=P\{X \leq t\}=\sum_{j=1}^{\infty} a_{j}\left\{q^{j-s_{j-1}} p^{s_{j-1}}\right\} .
$$

\section{Proof:}

Let, $t=\sum_{i=1}^{\infty} a_{i}\left(\frac{1}{2}\right)^{i}$ and $a_{k_{r}}$ be the $r^{\text {th }}$ non-zero element in the sequence $\left\{a_{i}\right\}, r=1,2, \cdots$. Let $t_{r}$ be the number having the binary representation $\left(a_{1}, a_{2}, \cdots a_{k_{r}}, 0,0, \cdots\right)$ and $t_{0}=0$. It is to be noted that $t_{r}$ is a finite binary terminating number. If $t$ is not a finite binary terminating number then the sequence $\left\{t_{r}\right\}$ increases to $t$. If $t$ is a finite binary terminating number then we note that $t=t_{r}$ for some finite $r$. For example, let $t$ have a binary representation $00010011000 \cdots$ and

$k_{1}=4, k_{2}=7, k_{3}=8, k_{4}=11$ so that $t_{1} \stackrel{B R}{\Leftrightarrow}(0010000 \cdots)$, $t_{2} \stackrel{B R}{\Leftrightarrow}(0001001000 \cdots), \quad t_{3} \Leftrightarrow(00010011000 \cdots)$ and so on. We note that $a_{i}=1$ for $i=k_{1}, k_{2}, k_{3}, \cdots$ and 0 otherwise. Note that

$$
\begin{aligned}
& P\left\{X=t_{r}\right\}= \\
& P\left\{Z_{i}=a_{i}, i=1,2, \cdots, k_{r}-1, Z_{k_{r}}=1, Z_{k_{r}+j}=0, j=1,2, \ldots\right\} \\
& \leq P\left\{Z_{k_{r}}=1, Z_{k_{r}+j}=0, j=1,2, \cdots\right\}=0 .
\end{aligned}
$$

Thus $F$ does not have a jump at $t_{r}$ and $F\left(t_{r}\right) \uparrow F(t)$.

Let $t$ be not a finite binary representing number. We note that

$$
\begin{gathered}
P\left(0 \leq X<t_{1}\right)=P\left(Z_{1}=0, \cdots, Z_{k_{1}}=0\right)=q^{k_{1}} \\
P\left(t_{1} \leq X<t_{2}\right)=P\left(Z_{1}=0, \cdots, Z_{k_{1}-1}=0, Z_{k_{1}}=1, Z_{k_{1}+1}=0,\right. \\
\left.\cdots, Z_{k_{2}}=0\right)=q^{k_{2}-1} p \\
P\left(t_{2} \leq X<t_{3}\right)=P\left(Z_{1}=0, \cdots, Z_{k_{1}-1}=0, Z_{k_{1}}=1, Z_{k_{1}+1}=0, \cdots\right. \\
\left.\cdots, Z_{k_{2}-1}=0, Z_{k_{2}}=1, Z_{k_{2}+1}=0, \cdots, Z_{k_{3}}=0\right)=q^{k_{3}-2} p^{2} .
\end{gathered}
$$

Thus in general for $r=1,2,3 \cdots$, we have

$$
P\left(t_{r-1} \leq X<t_{r}\right)=q^{k_{r}-(r-1)} p^{r-1} .
$$

Hence

$$
P(X<t)=\sum_{r=1}^{\infty} P\left(t_{r-1} \leq X<t_{r}\right)=\sum_{r=1}^{\infty} q^{k_{r}-(r-1)} p^{r-1} .
$$

If we let $k_{r}=j$, then $r-1=\sum_{i=1}^{j-1} a_{i}=s_{j-1}$, then we will have $P(X<t)=\sum_{j=1}^{\infty} a_{j}\left(q^{j-s_{j-1}} p^{s_{j-1}}\right)$.

In fact $F$ does not have jump at ant $t,(0<t<1)$.

Hence $P(X \leq t)=P(X<t)=\sum_{j=1}^{\infty} a_{j}\left(q^{j-s_{j-1}} p^{s_{j-1}}\right)$. 
However, if $t$ is a finite binary termination number then $t=t_{\mathrm{r}}$ for some finite number $r$ and

$$
\begin{aligned}
& P(X \leq t)=P(X<t)+P\left(X=t_{r}\right) \\
& =P\left(X<t_{r}\right)=\sum_{j=1}^{r} a_{j}\left(q^{j-s_{j-1}} p^{s_{j-1}}\right)=\sum_{j=1}^{\infty} a_{j}\left(q^{j-s_{j-1}} p^{s_{j-1}}\right)
\end{aligned}
$$

since $a_{j}=0, j=r+1, r+2, \cdots$.

\subsection{Particular Case}

If $p=\frac{1}{2}$, then $P(X<t)=\sum_{j=1}^{\infty} a_{j}\left(\frac{1}{2}\right)^{j}=t, t \in(0,1)$.

Hence $X \sim U(0,1)$.

\section{Remarks:}

In Fact it can be verified that (5) satisfies (2). It follows from the fact that if $t$ has binary representation $a=$ $\left(a_{1}, a_{2}, \cdots\right)$ then for

1) $0<t<1, t / 2$ has binary representation $u=\left(u_{1}, u_{2}, \cdots\right)$ where $u_{1}=0$ and $u_{i+1}=a_{i}, i=1,2, \cdots$ and

2) $1 / 2<t<1,2 t-1$ has binary representation $v=\left(v_{1}, v_{2}, \cdots\right)$ where $v_{i}=a_{i+1}, \mathrm{i}=1,2, \cdots$.

\section{Theorem 2:}

If $u$ and $v$ have binary representations $\left(a_{1}, a_{2}, \cdots, a_{n}, 0\right.$, $0, \cdots, 0)$ and $\left(a_{1}, a_{2}, \cdots, a_{n}, 1,1, \cdots, 1\right)$ respectively then the conditional distribution of $X$ given $u \leq X \leq v$ is that of $u+2^{-n} X$.

Proof: Follows by the definition of $X$.

\section{Theorem 3:}

Let $t \stackrel{B R}{\Leftrightarrow}\left\{a_{n}\right\}$ with $\left(a_{1}, a_{2}, \cdots, a_{k}, 0,0, \cdots\right)$ with $a_{k}=1$.

Then for $0 \leq s \leq \frac{1}{2^{k}}$

$$
P\left\{0<X-t<s \mid 0<X-t<\frac{1}{2^{k}}\right\}=P\left\{0<X<2^{k} s\right\} .
$$

\section{Proof:}

$$
\begin{aligned}
& P\left\{0<X-t<s \mid 0<X-t<2^{-k}\right\} \\
& =P\left\{0<X<t+s \mid 0<t<t+2^{-k}\right\}=\frac{P\{0<X<t+s\}}{P\left\{0<X<t+2^{-k}\right\}} \\
& =\frac{P\left\{Z_{1}=a_{1}, Z_{2}=a_{2}, \cdots, Z_{k}=a_{k}, \sum_{j=k+1}^{\infty}\left(\frac{1}{2}\right)^{j} Z_{j} \leq s\right\}}{P\left\{Z_{1}=a_{1}, Z_{2}=a_{2}, \cdots, Z_{k}=a_{k}\right\}} \\
& =\frac{P\left\{Z_{1}=a_{1}, Z_{2}=a_{2}, \cdots, Z_{k}=a_{k}\right\} P\left\{\left(\frac{1}{2}\right)^{k} \sum_{j=0}^{\infty}\left(\frac{1}{2}\right)^{j} Z_{j} \leq s\right\}}{P\left\{Z_{1}=a_{1}, Z_{2}=a_{2}, \cdots, Z_{k}=a_{k}\right\}} \\
& =P\left\{\left(\frac{1}{2^{k}}\right) X \leq s\right\}=P\left\{X \leq 2^{k} s\right\} .
\end{aligned}
$$

\section{Theorem 4:}

For $0 \leq t<s \leq \frac{1}{2}$ we have

$$
P\{t<X<s\}=\frac{q}{p} P\left\{t+\frac{1}{2}<X<s+\frac{1}{2}\right\} .
$$

\section{Proof:}

Let $Z=\left(Z_{1}, Z^{(2)}\right)=\left(Z_{1}, Z_{2}, Z_{3}, \cdots\right)$

$$
\begin{aligned}
& P\{t<X<s\}=P\left\{Z_{1}=0, Z^{(2)} \in A\right\} \\
& =P\left\{Z_{1}=0\right\} P\left\{Z^{(2)} \in A\right\}=q P\left\{Z^{(2)} \in A\right\}
\end{aligned}
$$

and

$$
\begin{gathered}
P\left\{t+\frac{1}{2}<X<s+\frac{1}{2}\right\}=P\left\{Z_{1}=1, Z^{(2)} \in A\right\} \\
P\left\{Z_{1}=1\right\} P\left\{Z^{(2)} \in A\right\}=p P\left\{Z^{(2)} \in A\right\} .
\end{gathered}
$$

In the above in fact $P\left\{Z^{(2)} \in A\right\}=P\left\{\frac{X}{2}<s-t\right\}$.

Hence the result.

\section{Theorem 5:}

If $F(., p)$ and $G(., p)$ are the distribution functions of $X$ and $1-X$ respectively then $G(x, p)=F(x, 1-p)$.

\section{Proof:}

If $X=\sum_{j=1}^{\infty}\left(\frac{1}{2}\right)^{j} Z_{j}$ and $Z_{j}$ 's are i.i.d. $B(1, p)$ then $1-X=\sum_{j=1}^{\infty}\left(\frac{1}{2}\right)^{j}\left(1-Z_{j}\right)=\sum_{j=1}^{\infty}\left(\frac{1}{2}\right)^{j} Y_{j}$ where $Y_{j}$ 's are i.i.d. $B(1,1-p)$.

Hence the result.

Mean and Variance of $X$ :

$$
\begin{gathered}
E(X)=\sum_{j=1}^{\infty}\left(\frac{1}{2}\right)^{j} E\left(Z_{j}\right)=\sum_{j=1}^{\infty}\left(\frac{1}{2}\right)^{j} p=p \\
E\left(X^{2}\right)=\sum_{j=1}^{\infty}\left(\frac{1}{2}\right)^{2 j} E\left(Z_{j}\right)+2 \sum_{i=1}^{\infty} \sum_{j=1}^{i-1}\left(\frac{1}{2}\right)^{i+j} E\left(Z_{i} Z_{j}\right) \\
=\sum_{j=1}^{\infty}\left(\frac{1}{2}\right)^{2 j} p+2 p^{2} \sum_{i=1}^{\infty}\left(\frac{1}{2}\right)^{i} \sum_{j=1}^{i-1}\left(\frac{1}{2}\right)^{j} \\
=\frac{p}{3}+2 p^{2} \sum_{i=1}^{\infty}\left(\frac{1}{2}\right)^{i}\left\{1-\left(\frac{1}{2}\right)^{i-1}\right\} \\
=\frac{p}{3}+2 p^{2}\left(\frac{1}{3}\right)=\frac{p(1+2 p)}{3}
\end{gathered}
$$

Hence $\operatorname{Var}(X)=\frac{p(1-p)}{3}$.

By using the c.f. $\Psi(t)=\prod_{k=1}^{\infty}\left[1-p+p \mathrm{e}^{i t / 2^{k}}\right]$ the cumulants can also be obtained. 


\section{Nonexistence of Density Function}

We have proved that the distribution function of $X$ is given by $F(t)=\sum_{j=1}^{\infty} a_{j}\left\{q^{j-s_{j-1}} p^{s_{j-1}}\right\}, t \Leftrightarrow\left\{a_{n}\right\}$. Let the left derivative and the right derivative of $F$ at $t$ exist. These be denoted by $f(t+)$ and $f(t-)$ respectively. Consider $f\left(\frac{1}{2}+\right)$ and $f\left(\frac{1}{2}-\right)$, the right and left derivatives of $F$ at $1 / 2$.

$$
\begin{aligned}
f\left(\frac{1}{2}-\right) & =\lim _{k \rightarrow \infty} \frac{F\left(\frac{1}{2}\right)-F\left(\frac{1}{2}-\frac{1}{2^{k}}\right)}{2^{-k}} \\
& =\lim _{k \rightarrow \infty} \frac{q p^{k-1}}{2^{-k}}=\lim _{k \rightarrow \infty}(2 q)(2 p)^{k-1}
\end{aligned}
$$

and

$$
\begin{aligned}
f\left(\frac{1}{2}+\right) & =\lim _{k \rightarrow \infty} \frac{F\left(\frac{1}{2}+\frac{1}{2^{k}}\right)-F\left(\frac{1}{2}\right)}{2^{-k}} \\
& =\lim _{k \rightarrow \infty} \frac{p q^{k-1}}{2^{-k}}=\lim _{k \rightarrow \infty}(2 p)(2 q)^{k-1} .
\end{aligned}
$$

Note that $f\left(\frac{1}{2}-\right)$ and $f\left(\frac{1}{2}+\right)$ are equal if and only if $p=q=\frac{1}{2}$. Hence $F$ is not differentiable at $1 / 2$ if $p \neq \frac{1}{2}$.

Let $D=\left\{\frac{r}{2^{k}}: k=1,2,3 \cdots, r=0,1,2, \cdots, 2^{k}\right\}$. It can be shown that $F$ is not differentiable at each point of $D$ and the set $D$ is countable dense subset of $[0,1]$. Hence $F$ is nowhere differentiable in $[0,1]$.

\section{Distribution of Sum of a Finite Number of Bernoulli Random Variables}

Since $F(t)$ is an infinite series for $t$ not in $D$, the exact evaluation of $F(t)$ is not possible for each $t \in(0,1)$. More- over, the density function of $X$ does not exist on the interval $(0,1)$. Hence in the following we consider the sequence $\left\{X_{\mathrm{k}}\right\}$ of r.v. defined by

$$
X_{k}=\sum_{i=1}^{k}\left(\frac{1}{2}\right)^{i} Z_{i} .
$$

The sequence $\left\{X_{k}\right\}$ increases point wise to $X$ and $X-X_{k}<2^{-k}$.

Similar to (5), it can be shown that for $t=\sum_{j=1}^{\infty}\left(\frac{1}{2}\right)^{j} a_{j}$, the d.f. of $X_{k}$ is $F_{k}(t)=\sum_{j=1}^{k} a_{j}\left\{q^{j-s_{j-1}} p^{s_{j-1}}\right\}=F(s)$, where $s=\sum_{j=1}^{k} a_{j}\left(\frac{1}{2}\right)^{j}$. Further, at these values of $s$, $F_{k}(s)=F(s)$ and the difference between two successive values of $s$ 's is $2^{-k}$, as such the two functions $F_{k}(t)$ and $F(t)$ are almost alike. Since the sequence $\left\{X_{k}\right\}$ increases point wise to $X$ the sequence $\left\{F_{k}(t)\right\}$ decreases to $F(t)$ for $t \in(0,1)$.

We note that for $p=1 / 2, F_{k}(t)=F(s)$. The graphs of p.m.f and d.f. of $X_{5}$ and $X_{10}$ for different values of $p \neq \frac{1}{2}$ are given in the Appendix.

\section{Acknowledgements}

We are thankful to Professor H. J. Vaman, Central University of Rajasthan, India, for the discussions which helped to improve the content and the presentation of the paper.

\section{References}

[1] S. Kunte and R. N. Rattihalli, "Uniform Random Variable. Do They Exist in Subjective Sense?" Calcutta Statistical Association Bulletin, Vol. 42, 1992, pp. 124-128.

[2] K. L. Chung, "A Course in Probability Theory," 3rd Edition, Academic Press, Cambridge, 2001. 


\section{Appendix}

The graphs of p.m.f and d.f. of $\boldsymbol{X}_{\boldsymbol{k}}$ for different values of $\boldsymbol{p}$.

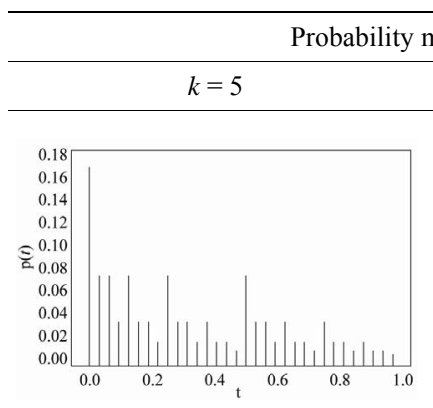

mass function
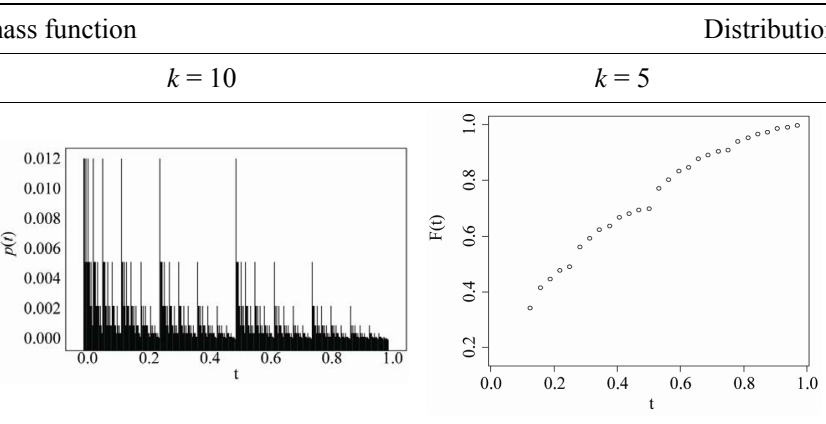

Distribution function

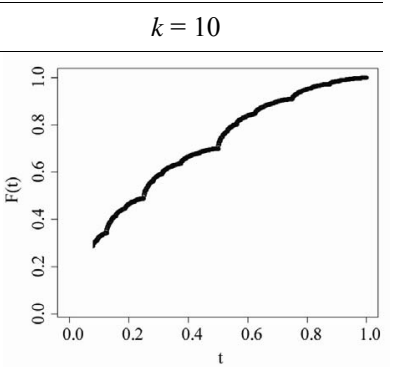

$p=0.3$
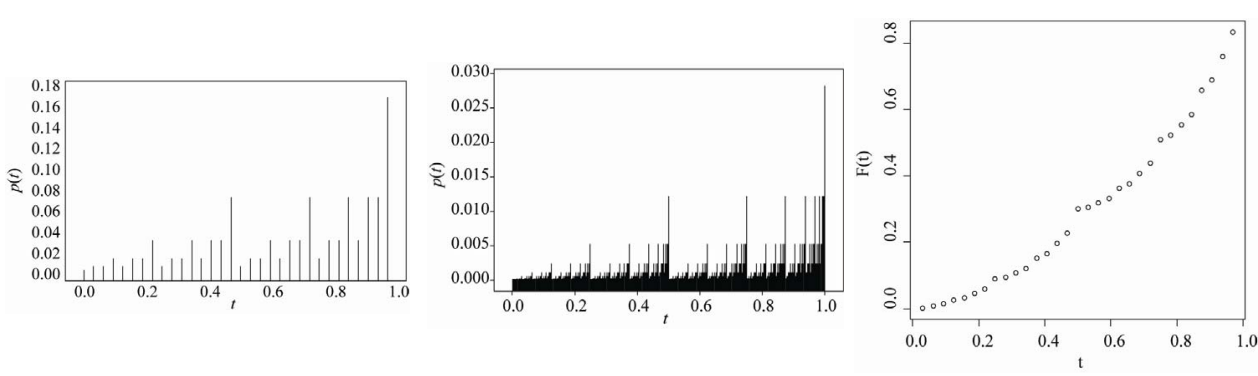

$p=0.3$

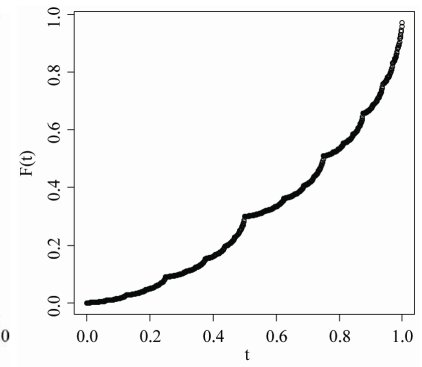

$p=0.7$

$p=0.7$
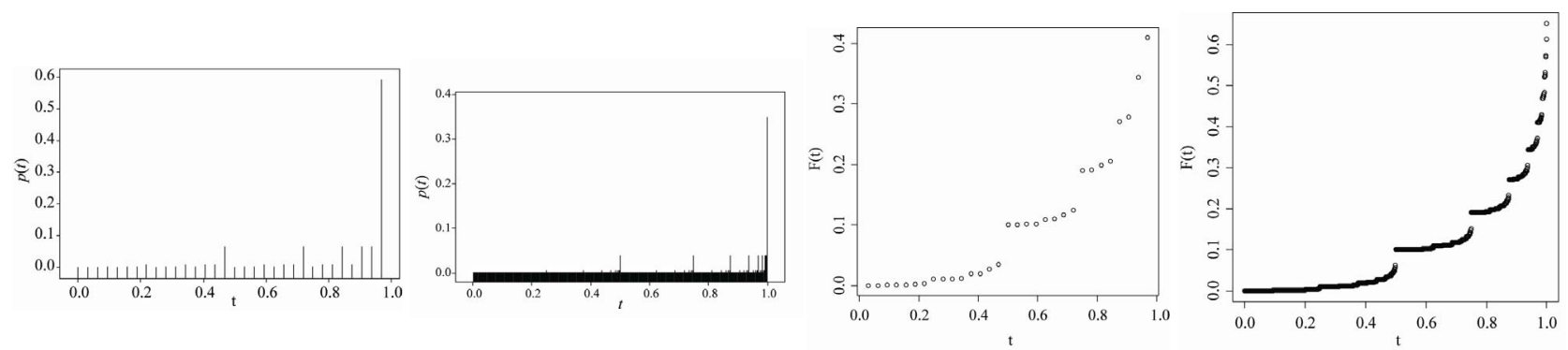